\title{
Orthotopic Animal Model of Pseudomyxoma Peritonei
}

\section{An in Vivo Model to Test Anti-Angiogenic Drug Effects}

\author{
Anthony Dohan, ${ }^{* \dagger}$ Ruben Lousquy, ${ }^{*}$ Clarisse Eveno, ${ }^{* \ddagger}$ Diane Goere, ${ }^{\S}$ Dong Broqueres-You, ${ }^{* \llbracket}$ Rachid Kaci, ${ }^{* \|}$ \\ Jacqueline Lehmann-Che, ${ }^{* *}$ Jean-Marie Launay, ${ }^{* \dagger}$ Philippe Soyer, ${ }^{* \dagger}$ Philippe Bonnin, ${ }^{* \ddagger}$ and Marc Pocard ${ }^{* \ddagger}$
}

\begin{abstract}
From INSERM U965, Carcinomatosis Angiogenesis and Translational Research (C.A.R.T.), * the Departments of Body and Interventional Imaging, ${ }^{\dagger}$ Pathology, Biochemistry, ${ }^{\dagger \dagger}$ and Clinical Physiology-Functional Investigations, ${ }^{\ddagger}$ and the Surgical Oncologic and Digestive Unit ${ }^{\ddagger}$ Lariboisière Hospital, Paris Diderot University, Sorbonne Paris Cité, Assistance Publique - Hôpitaux de Paris, Paris; the Department of Surgical Oncology, ${ }^{\S}$ Gustave Roussy Institute, Villejuif; the Blood and Vessels Institute, "Paris Diderot University, Sorbonne Paris Cité, Lariboisière Hospital, Paris; and the Department of Biochemistry and INSERM U944, ** Paris Diderot University, Sorbonne Paris Cité, Assistance Publique - Hôpitaux de Paris, Saint-Louis Hospital, Paris, France
\end{abstract} CME Accreditation Statement: This activity ("ASIP 2014 AJP CME Program in Pathogenesis") has been planned and implemented in accordance with the Essential Areas and
policies of the Accreditation Council for Continuing Medical Education (ACCME) through the joint sponsorship of the American Society for Clinical Pathology (ASCP) and the
American Society for Investigative Pathology (ASIP). ASCP is accredited by the ACCME to provide continuing medical education for physicians.

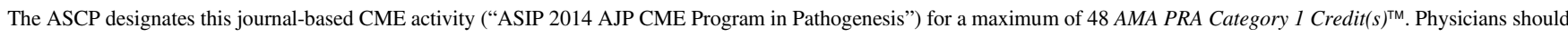
only claim credit commensurate with the extent of their participation in the activity.

CME Disclosures: The authors of this article and the planning committee members and staff have no relevant financial relationships with commercial interests to disclose.

Accepted for publication

March 6, 2014.

Address correspondence to Anthony Dohan, M.D., Department of Body and Interventional Imaging, Lariboisière Hôpital, Paris Diderot University 7 and INSERM UMR 965, 2 Rue Ambroise Paré, 75010 Paris, France. E-mail: anthony. dohan@1rb.aphp.fr.
Pseudomyxoma peritonei (PMP) is an uncommon peritoneal mucinous carcinomatosis confined to the peritoneal cavity. The rarity of PMP in humans makes evaluation of the disease biological features and new therapeutic strategies difficult. Accordingly, there is a need for animal models of PMP. Human PMP tissue was i.p. grafted and grown into nude mice, then constituted into reliable and reproducible orthotopic models. Histological and immunostaining analysis was performed. Bevacizumab was injected twice a week either during tumor growth or after cytoreductive surgery. In vivo imaging of tumor angiogenesis was performed using barium sulfate or isolectin microangiography and Doppler ultrasonography of the superior mesenteric artery. Tumor angiogenesis was confirmed by the presence of tortuous vascular networks with high levels of expression of CD31, vascular endothelial cadherin, and desmin. Doppler ultrasonography of the superior mesenteric artery revealed a twofold increase in blood flow velocity compared with tumor-free mice $(P<0.001)$. Bevacizumab administration was correlated with the normalization of tumor vascularity when injected during tumor growth and with the stabilization of the histological and hemodynamic findings when injected after cytoreductive surgery. Our PMP models mimic human PMP. Our results confirmed the presence of tumor angiogenesis related to PMP growth. Our murine model allows researchers to actually bench test and evaluate, in preclinical studies, the efficacy of new therapeutic strategies and anti-angiogenic therapies. (Am J Pathol 2014, 184: 1920-1929; http://dx.doi.org/10.1016/j.ajpath.2014.03.004)
Pseudomyxoma peritonei (PMP) is a rare malignant disease characterized by the progressive accumulation of mucinous tumor tissue in the peritoneal cavity without extraperitoneal growth, leading to intra-abdominal compression and a fatal outcome. The consensus is that PMP has an intestinal origin and results from the perforation of a mucinous appendiceal neoplasm. $^{1-4}$ Complete cytoreductive surgery with hyperthermic i.p. chemotherapy is now a well-established treatment option for patients with PMP. ${ }^{5-10}$ Despite the improved survival rate achieved with the combined treatment, this therapeutic option is not applicable to all patients, especially for those with coexisting causes of

Supported in part by a NORD Foundation (National Organization for Rare Disorders) grant, a Fondation de France grant, and an Association for Cancer Research personal grant (R.L.).

A.D. and R.L. contributed equally to this work.

P.B. and M.P. contributed equally to this work as senior authors.

Disclosures: None declared. 
morbidity. Moreover, treatment failure and recurrence are not rare. ${ }^{11}$

Histopathologically, PMP is graded from disseminated peritoneal adenomucinosis (DPAM or low grade) to peritoneal mucinous carcinomatosis (PMCA or high grade), with an intermediate histological type. ${ }^{12}$ PMP presents as large patches of mucinous deposits that contain a few cells organized as a lowproliferative epithelium. The paucity of tumor cells may explain the relative lack of efficacy of the common cytotoxic chemotherapy regimens on PMP. To date, we are aware of only two case reports that have studied the potential impact of an anti-angiogenic agent (ie, bevacizumab) in PMP and reported a partial response. ${ }^{13,14}$ To improve the overall survival and recurrence-free survival rate, the development of new therapeutic strategies that combine chemotherapy and/or an anti-angiogenic drug with cytoreductive surgery is of major importance.

Animal models of PMP should mirror the peritoneal growth pattern of PMP in humans and should also mirror the histopathological classification used in humans. In addition, such animal models should be appropriate for testing new anti-angiogenic therapies and evaluating the tumor response. To our knowledge, there are only three published animal studies that describe the development of orthotopic xenografted PMP in animal models. ${ }^{15,16}$

To date, the evaluation of patients with PMP is based on computed tomography and no functional imaging modality has a proven efficacy in the follow-up of these patients. Doppler ultrasonography (DUS) has been successfully used to monitor the tumor blood supply in primary and secondary liver cancers. ${ }^{17-21}$ The main blood supply of the PMP tumor arises from the superior mesenteric artery (SMA). We, thus, hypothesized that DUS of the SMA would provide an evaluation of the blood flow supplying the tumor vascular network. Indeed, it has been previously demonstrated that measurement of the blood flow velocity in the feeding artery upstream of the tumor vascular network allows for the semiquantitative analysis of the development or the involution of the tumor vasculature. ${ }^{17,21,22}$

The goals of our study were threefold. First, we wanted to develop a murine model of PMP to investigate tumor angiogenesis in PMP. Second, we wanted to evaluate the capabilities of DUS in depicting and quantifying the development or the involution of the tumor vasculature in this experimental murine model. Third, we wanted to investigate the effects of anti-angiogenic therapies alone or as adjuvant treatments of PMP after incomplete surgical resection.

\section{Materials and Methods}

\section{Orthotopic Murine Model of PMP}

\section{Patients}

All of the tissue samples were collected after Institutional Review Board approval and after informed consent was obtained from all of the patients. Tumor samples were obtained from 18 patients with histologically proven PMP that originated from an appendiceal mucinous neoplasm; patients were treated with surgery at two different institutions between November 2009 and May 2012.

\section{Animals}

Five-week-old female athymic Swiss nude mice (Charles River Laboratories International Inc., Arbresle, France), weighing between 16 and $18 \mathrm{~g}$, were acclimatized for 1 week before tumor transplantation. The animals were maintained under specific pathogen-free conditions, and irradiated food and water were supplied ad libitum. During surgical procedures, the animals were anesthetized with a mixture of $80 \mathrm{mg} / \mathrm{kg}$ ketamine and $16 \mathrm{mg} / \mathrm{kg}$ xylazine delivered i.p. (Virbac, Carros, France). All of the experimental protocols and the animal procedures for the care and use of laboratory animals were conducted according to the guidelines of our institution and the Federation of European Laboratory Animal Science Association.

\section{Tumor Collection and Implantation}

The tumor samples were collected and immediately brought to the animal facility. The fresh tumor tissue was cut into $3 \times$ $3 \times 3$-mm pieces. The tumor fragments were i.p. implanted in two mice for each sample. A 1-cm midline laparotomy was performed, and six tumor pieces were placed in the peritoneal cavity in both sides of the subdiaphragmatic area, the flank, and the pelvis. After implantation, the peritoneum and muscle were closed with one layer of Monocryl 5/0 (Ethicon, Somerville, NJ), and the skin was closed with staples.

\section{Sampling and Evaluation}

The mice were carefully monitored and weighed twice a week. The mice were sacrificed when they weighed $>40 \mathrm{~g}$. After sacrificing the mice, the macroscopic assessment of PMP was performed, during which a search for primary and metastatic tumors was performed; these tumors were sampled for further analyses. The tumor samples were embedded in optimal cutting temperature compound or fixed in formalin.

\section{Cytoreductive Surgical Procedure}

Cytoreductive surgery was performed at $33 \mathrm{~g}$. Each mouse was anesthetized with $1.5 \%$ isoflurane in $100 \% \mathrm{O}_{2}$. A large midline incision was made, and maximum resection was performed with a clip applier. Lesions adjacent to the digestive tract were not resected because of the high risk of a lethal wound. The peritoneum and muscle were closed with one layer of Monocryl 5/0, and the skin was closed with staples. Immediately after surgery, mice were given an analgesic drug (buprenorphine; Schering-Plough Europe, Brussels, Belgium) in a single s.c. dose of $0.1 \mathrm{mg} / \mathrm{kg}$.

\section{Ex Vivo and in Vivo Characterization of Orthotopic Murine Models}

\section{Histological and Immunohistological Analyses}

Tissue from the mouse tumor was explanted after sacrifice, fixed with formalin, and stained with H\&E-safran and Alcian Blue. Histological classification was performed according to the three 
groups, as described by Winer and Buckanovich ${ }^{14}$ : 1 , DPAM; 2, PMCA; and 3, intermediate histological type PMCA.

Archival paraffin-embedded tissue blocks were cut into sections (4 to $5 \mu \mathrm{m}$ ) and transferred to glass slides. After deparaffinization, antigen retrieval was performed using citrate solution at $\mathrm{pH}$ 6.0. The slides were stained on an automated Benchmark Ultra immunostainer (Ventana Medical Systems, Tucson, AZ). The monoclonal anti-intestinal mucin (MUC)-2 antibody (cloner Ccp58; Novocastra, Newcastle, UK) was used at 1:100 dilutions. The epitope specifically recognized by the anti-MUC-2 antibody is human MUC-2 glycoprotein (no cross-reaction with MUC-1- or MUC-3-derived peptides).

Slides were incubated with the previously described primary antibody for 1 hour at room temperature; thereafter, goat anti-mouse secondary antibody was applied, and the reaction was developed using diaminobenzidine.

In addition, tumor samples from each passage through passage 5 were embedded in optimal cutting temperature compound for the preparation of frozen tissue sections $(5 \mu \mathrm{m}$ thick), which were then fixed in acetone at $4^{\circ} \mathrm{C}$ for 10 minutes and immunostained as described. After washing, the nonspecific sites were saturated for 20 minutes with bovine serum albumin (10 g/L in PBS-1\% Tween). The sections were then incubated for 1 hour with the following primary antibodies: anti-pan-cytokeratin antibody (1:100; Abcam, Cambridge, MA) and anti-cytokeratin 20 (CK20) antibody (prediluted) (Abcam), which are specific for colon cancer; an antibody against Ki-67 (1:100; Neomarker Inc., Fremont, CA), which is a nuclear protein expressed in proliferating cells; antibodies against platelet endothelial cell adhesion molecule (CD31) (1:50; BD Pharmingen, Le Pont-de-Claix, France); an antibody against vascular endothelial (VE) cadherin (1:50; Santa Cruz Biotechnology Tebu SA, Le Perray-en-Yvelines, France), which is a protein localized at the intercellular junctions of endothelial cells; and an antibody against desmin (1:200; Neomarker Inc.), which is a protein specific for smooth and striated muscle. After washing, the sections were incubated for 30 minutes with the appropriate secondary antibody (1:200) [Alexa Fluor 555 goat anti-mouse, goat antirabbit, or donkey anti-goat, or Alexa Fluor 488 goat antimouse, donkey anti-rat, or donkey anti-goat (Interchim, Asnieres, France)] and then washed with PBS-1\% Tween. The primary antibody was omitted on sections that were used as negative controls. To perform double immunostaining, the sections were initially incubated with the first primary antibody, then with the second primary antibody, and, finally, with the appropriate secondary antibody. The histological sections were analyzed with a fluorescence microscope equipped with the appropriate filters (Observer.Z1; Carl Zeiss MicroImaging GmbH, Göttingen, Germany).

\section{TP53 Functionality and KRAS Mutation Analysis}

Mutational analysis of TP53 was performed using functional analysis of separated alleles in yeast, in accordance with the method described by Flaman et al. ${ }^{23}$
This method requires frozen tumor samples and evaluates the transactivation activity of $\mathrm{p} 53$ on a p53-responsive promoter stably integrated in the yeast genome. RNA was extracted by the phenol-chloroform method and reverse transcribed, and p53 transcripts were amplified by PCR and transfected into yeast. Yeast colonies transformed with wildtype or mutated TP53 sequences appear white and large or red and small, respectively. TP53 status was considered mutated when $>10 \%$ of the yeast colonies were red and analysis using the split versions of the test could identify the defect in the $5^{\prime}$ or $3^{\prime}$ part of the gene.

The seven most frequent KRAS mutations on codons 12 and 13 were analyzed by allelic discrimination after DNA extraction from selected formalin-fixed, paraffin-embedded sections ( $>20 \%$ tumor cells) of the tumor samples using the QIAamp DNA minikit (Qiagen, Hilden, Germany), according to manufacturer's instructions. Specific probes for each allele (mutated or not) were labeled with fluorescent reporter dyes at their $5^{\prime}$ end and analyzed by real-time PCR on an LC480 instrument (Roche Diagnostics, Meylan, France). ${ }^{24}$

\section{Microangiography}

Conventional microangiography was performed in five grafted mice to characterize the tumor vascular network. Under anesthesia, a longitudinal laparotomy was performed, and the abdominal aorta was exposed by moving the tumor to the left side and then ligating upstream of the iliac bifurcation. A polyethylene catheter with monitored pressure and volume was introduced into the abdominal aorta to inject a contrast material (barium sulfate, $1 \mathrm{~g} / \mathrm{mL}$ ). Image acquisition was performed with a digital X-ray transducer (model 2100; Kodak Dental Systems, Atlanta, GA). ${ }^{22}$

Alternatively, performing the same surgical procedure in another five mice, we perfused $0.5 \mathrm{~mL}$ of heparinized saline and $0.8 \mathrm{~mL}$ of bandeiraea simplicifolia isolectin B4 (IB4) conjugated to fluorescein isothiocyanate (Sigma, Saint Quentin Fallavier, France), diluted with PBS to a concentration of $0.25 \mathrm{mg} / \mathrm{mL}$. This tracer binds selectively to terminal $\alpha$-galactose (IB4) ${ }^{25}$ and is an endothelial cell-specific marker that was used to identify and characterize the neovessels in the tumor. Fifteen minutes later, the mice were sacrificed, and the tumors were harvested. After fixation, the tumor samples were analyzed with a computer-assisted Nikon fluorescence microscope with a digital camera (Eclipse TE200; Nikon Inc., Melville, NY). Because of the heterogeneous distribution of the vasculature and the presence of wide ranges of mucinous content, we did not quantify the density of the vessels.

\section{Ultrasonographic Examination}

Ten Swiss, nude, PMCA tumor-grafted and 10 age-matched normal mice had ultrasonographic measurements using a DUS instrument (Vivid 7; GE Medical Systems Ultrasound, Horten, Norway) equipped with a 12-MHz linear transducer, as previously described. ${ }^{22}$ Briefly, after sedation, the mice were placed on a heating blanket $\left(38^{\circ} \mathrm{C}\right)$ in the left lateral prone position to avoid placing pressure on the 
abdomen with the transducer. The color Doppler mode was activated with an anterior longitudinal view of the abdomen crossing the longitudinal axis of the aorta. The SMA and celiac trunk were localized on the screen by their colorcoded blood flow. A pulsed Doppler sample was then placed on the longitudinal axis of each vessel, and pulsed Doppler velocity waveforms were recorded. Peak-systolic, end-diastolic, and time-averaged mean blood flow velocities (BFVs) were measured according to the pulsed Doppler spectrum. The DUS recordings were repeated every 2 weeks from the initiation of the tumor graft up to 6 weeks.

\section{Serum Angiogenesis Markers}

After sacrificing the mice, blood was collected into chilled tubes containing heparin (Sarstedt, Marnay, France). The plasma concentrations of vascular endothelial growth factor (VEGF), placental growth factor (PIGF), and transforming growth factor $\beta$ (TGF- $\beta$ ) were determined with ELISA tests (MMV00, DPG00, and MB100B, respectively), according to the manufacturer's instructions.

\section{Treatment}

In another set of experiments, we applied an anti-angiogenic treatment in two models of PMCA (PMCA-1 and PMCA-2). Four weeks after the PMCA surgical graft, the mice were randomly assigned to two different groups. The mice in the treated groups were i.p. injected twice a week with $5 \mathrm{mg} / \mathrm{kg}$ bevacizumab ( $n=10$ for PMCA-1 and $n=10$ for PMCA-2), according to prior publications. The mice in the control groups ( $n=10$ for each experiment) received an equivalent volume of PBS. The mice were sacrificed when they weighed $>40 \mathrm{~g}$. The serum angiogenesis markers (VEGF, PlGF, and TGF- $\beta$ ) were quantified in these two groups 6 weeks after the tumor graft and compared with five age-matched, non-grafted mice. In another set of experiments, after cytoreductive surgery was performed at the weight of $33 \mathrm{~g}$, the grafted mice were submitted to an anti-angiogenic treatment with bevacizumab $(n=$ 11) versus PBS $(n=9)$, i.p. injected twice a week in an adjuvant setting. The mice were carefully monitored for 4 weeks with DUS and weighed weekly. The development of tumor growth was evaluated by following the increase in weight. Serum angiogenesis markers were also quantified.

\section{Statistical Analysis}

Results were expressed as the means \pm SD. Heart rate, blood flow velocities, and concentrations of serum angiogenesis markers were compared using an analysis of variance. Post hoc paired and unpaired Student's $t$-tests were performed to identify which time point or which group differences accounted for the significant analysis of variance. The survival probabilities were estimated using the Kaplan-Meier product-limit method (MedCalc, version 10.4.3.0; MedCalc Software, Ostend, Belgium). $P<0.05$ was considered to be significant.

\section{Results}

\section{Tumor Growth in the Animal Models}

Among the 18 i.p. grafted, patient-retrieved PMP samples, nine succeeded in growing, but growth occurred only once for one of the samples. Among the eight remaining samples, seven were similar to a PMCA (PMCA-1 to PMCA-7), and one was representative of a DPAM. These eight models were considered to be reliable and reproducible orthotopic mouse models of PMP. The delay between successive tumor growths decreased from 136 days (range, 116 to 166 days) after the first transplant to 78 days (range, 42 to 115 days) after the fifth transplant. The take rates ranged from $80 \%$ to $100 \%$ for PMCA-1 to PMCA-7 and from $40 \%$ to $90 \%$ for DPAM. As observed in humans, the mice presented abdominal distension due to tumor growth. PMCA-1 to PMCA-7 were considered as more aggressive compared with DPAM because these tumors grew more quickly.

The typical initial i.p. growth pattern was characterized by mucinous ascites, accompanied by a variable number of solid mucinous tumor lesions of varying sizes. The solid tumor components were attached to the peritoneum and serosa of all of the i.p. organs. No animal presented any metastatic lesions.

\section{Microscopic Assessment}

The animal models of PMP reflect the histopathological classification after each transplant generation. In the mice, we confirmed with H\&E-safran staining that DPAM developed from a low-grade appendiceal mucinous tumor. This subtype was characterized by widespread peritoneal deposits of abundant pools of extracellular mucin with strips of proliferative mucinous columnar epithelium with little cytological atypia or mitotic activity (Supplemental Figure S1, A and B). PMCA consisted of a juxtaposition of glands formed by an abundant mucinous epithelium and/or signet ring cells and commonly had severe cytological atypia. This subtype had poorly differentiated architecture (Supplemental Figure S1, C and D). The pathological characteristics were conserved through the successive tumor passages, with no change in the proportion of mucinous ascites and solid mucinous tumor lesions.

High levels of expression of pan-cytokeratin and CK20, as indicated with immunostaining, were observed in the tumor through the successive tumor passages from mouse to mouse, which indicates that the human and digestive origin was maintained (Supplemental Figure S2, A and B). Low levels of DAPI immunostaining and the presence of only a few isolated Ki-67-positive tumor cells were observed, which verified the paucicellular and low proliferative characteristics of the tumor (Supplemental Figure S2, C and D).

Immunohistochemistry showed high levels of cytoplasmic staining of tumor cells by the antibody anti-MUC2 and in the human tumor as after passages from mouse to mouse, confirming that tumor kept its biochemical 
characteristics with a high production of mucin (Supplemental Figure S3, A and B).

Molecular characteristics were also analyzed in the PMCA-2 model for initial patient and xenografted PMP in two mice. No alteration of the p53 functionality (TP53 wild type) was detected in the initial human sample or in the xenografted tumors. Nevertheless, an activating p.G12V mutation of the KRAS gene was detected in the human sample and two xenografted mice, highlighting that the xenografted tumors reflect the human origin at the molecular level.

\section{Angiogenesis Analysis}

Tumor angiogenesis was visualized with anti-CD31 and anti-VE-cadherin antibodies, which are specific for endothelial cells (Supplemental Figure S2, E-G), and an antidesmin antibody, which stained pericytes (Supplemental Figure $\mathrm{S} 2 \mathrm{H}$ ). Double staining confirmed the colocalization of the VE-cadherin and desmin immunoreactivity.

\section{Microangiography}

After in vivo intra-aortic injection of barium sulfate (Figure 1A) or IB4 (Figure 1B), microangiography revealed the arterial vascular network and showed the abdominal aorta, the left and right renal arteries, the gastric arteries, the hepatic arteries, and the superior mesenteric artery. The vascular networks downstream of the SMA presented many functional tortuous and irregular newly developed vessels going toward and through the i.p. tumor.

\section{Ultrasonographic Examination}

The tumor growth and development of ascites were observed by DUS (Figure 2A). In the control mice, time-averaged mean BFV measurements in the SMA and in the celiac trunk remained stable: $6.9 \pm 1.9 \mathrm{~cm} / \mathrm{second}$ and $12.5 \pm 4.4 \mathrm{~cm} / \mathrm{second}$, respectively (Figure 2, B-E and F). In contrast, in the tumor group, the mean BFV in the SMA increased from the time of the tumor graft to the time of sacrifice (from $6.9 \pm 1.9$ to $13.8 \pm 2.1$ $\mathrm{cm} / \mathrm{second} ; P<0.001$ ) (Figure 2, $\mathrm{C}$ and $\mathrm{E}$ ), although the mean $\mathrm{BFV}$ remained stable in the celiac trunk (Figure 2, D and F). The 1.75-fold increase in the mean BFV in the SMA compared with the control values $(P<0.001)$ was representative of the development of the vascular network downstream from the artery (ie, the number of opened and dilated vessels, thus indicating tumor angiogenesis of the peritoneal tumor).

\section{Treatments}

Treatment with an Anti-Angiogenic Agent (Bevacizumab) Alone without Cytoreductive Surgery

Survival was defined by the time required to reach $40 \mathrm{~g}$ weight. Mice in the PMCA-1-grafted and PMCA-2grafted mouse models, treated with bevacizumab, had a significantly greater survival time than the mice in the control groups without treatment (Figure 3, A and B). For the PMCA-1 model, the median survival time was 79.8 days (range, 45 to 103 days) in the treated group $(n=10)$ and 58.4 days (range, 45 to 68 days) in the control group ( $n=$ 10) $[P<0.001$; hazard ratio (HR), $0.12 ; 95 \% \mathrm{CI}, 0.03$ to 0.47]. For the PMCA-2 model, the median survival time

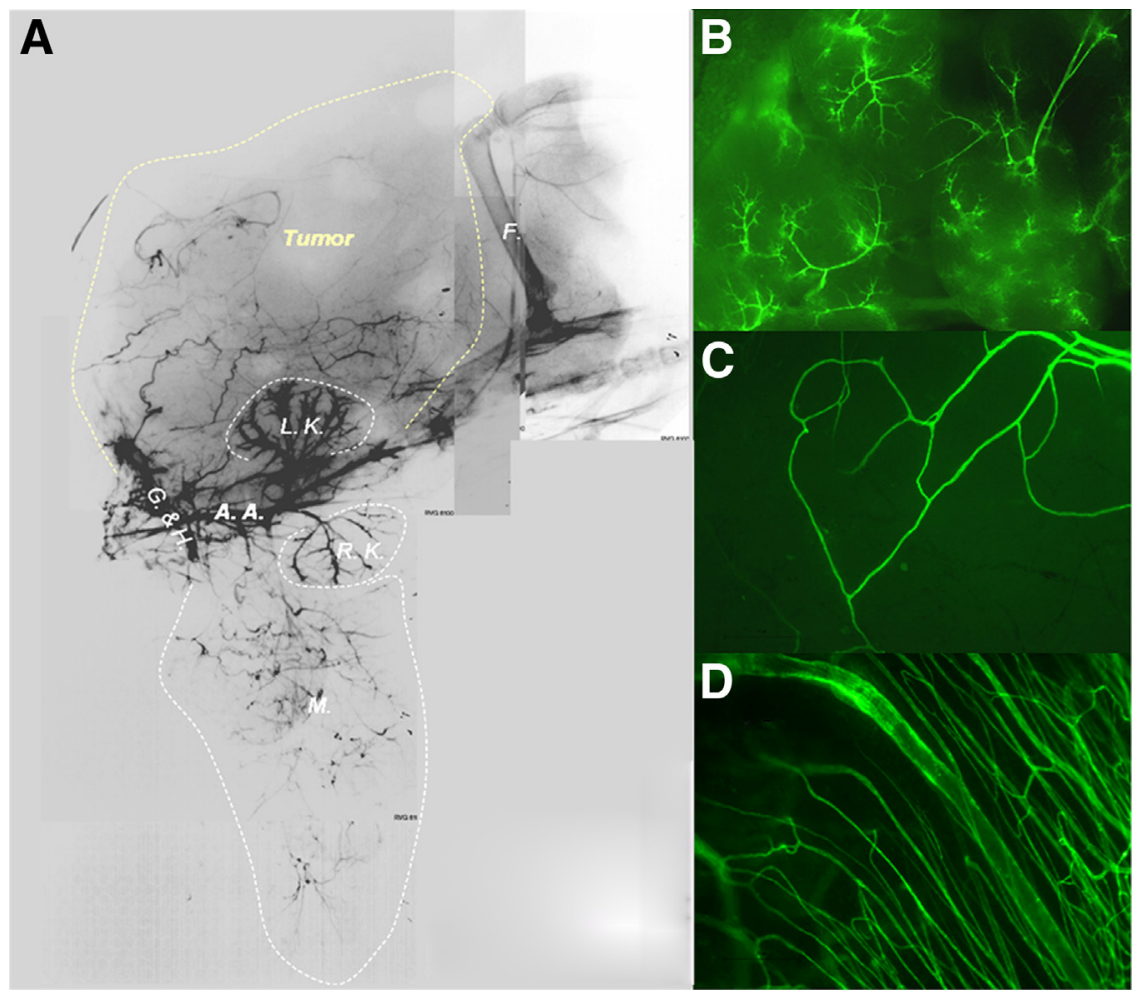

Figure 1 A: Representative computed photomicroangiography performed with a digital $X$-ray transducer in a 12-week-old mouse with PMP. B: Representative images of neoangiogenesis in PMP after in vivo intra-aortic injection of IB4 (green fluorescence) in untreated control mice. $\mathbf{C}$ and $\mathbf{D}$ : After 6 weeks of bevacizumab treatment, the tumor vessels appeared more regular, straight, and organized. Original magnification: $\times 10$ (B-D). A.A., abdominal aorta; F., femur; G.\&H., gastric and hepatic; L.K., left kidney; M., mesenteric; R.K., right kidney. 

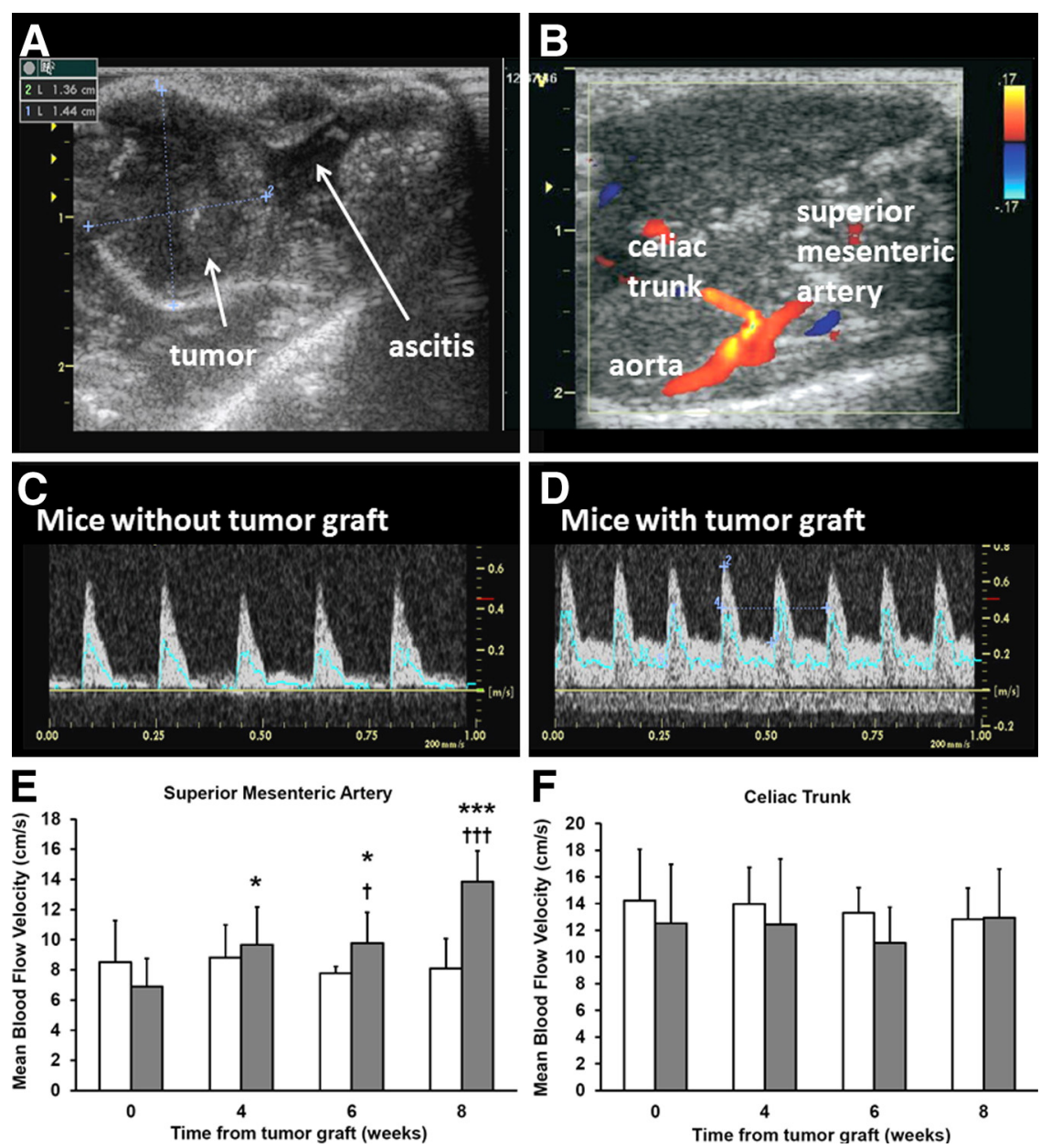

Figure 2 A: Two-dimensional DUS of the abdomen of a 12-week-old mouse with PMP showing solid tumor and ascites. B: Anterior longitudinal view of the abdomen crossing the longitudinal axis of the aorta, with the SMA and celiac trunk revealed by color-coded Doppler ultrasonography. Representative BFV waveforms recorded in the SMA in ungrafted (C) and PMP-grafted (D) mice. The blue line represents the time-averaged mean BFV. $E$ and $\mathbf{F}$ : Changes in the mean BFV in the SMA and in the celiac trunk from the time of the tumor graft to 6 weeks afterward $(n=10)$. The mean BFV remained constant in the celiac trunk but increased markedly in the SMA. ${ }^{*} P<0.05,{ }^{* * *} P<0.001$ versus the beginning of the recordings just after tumor graft; ${ }^{\dagger} P<0.05,{ }^{\dagger \dagger \dagger} P<0.001$ versus healthy mice $(n=10)$. Gray bars, PMP; white bars, controls. was 81.6 days (range, 54 to 126 days) in the treated group $(n=9)$ and 58.2 days (range, 51 to 70 days) in the control group ( $n=9 ; P=0.002 ; \mathrm{HR}, 0.16 ; 95 \% \mathrm{CI}, 0.05$ to 0.57 ).

The plasma concentrations of VEGF, PlGF, and TGF- $\beta$ in the five healthy ungrafted mice were $17.9 \pm 2.7,20.9 \pm 4.1$, and $4.5 \pm 2.9 \mathrm{ng} / \mathrm{L}$, respectively. In the PMCA-1 model, the control mice $(n=5)$ presented dramatically increased plasma concentrations of $140.7 \pm 19.5,203.4 \pm 55.2$, and $28.1 \pm 5.4 \mathrm{ng} / \mathrm{L}$ for VEGF, PlGF, and TGF- $\beta$, respectively $(P<0.001$, compared with the healthy mice). In the bevacizumab-treated mice $(n=5)$, the serum levels were lower compared with the untreated grafted mice, and were decreased to $50.6 \pm 5 \mathrm{ng} / \mathrm{L}(P=0.005), 54.2 \pm 13.4 \mathrm{ng} / \mathrm{L}$ $(P=0.014)$, and $14.1 \pm 3.5 \mathrm{ng} / \mathrm{L}(P=0.047)$, respectively, for VEGF, PIGF, and TGF- $\beta$. However, the serum levels remained elevated compared with the healthy mice $(P<0.01)$ (Figure 4). After treatment with bevacizumab, the vessels were more organized, regular, and straight, as indicated by microangiography using IB4 in conjunction with fluorescein isothiocyanate (Figure 1, C and D).

\section{Treatment with an Anti-Angiogenic Agent (Bevacizumab)} after Cytoreductive Surgery

After surgery, all of the mice presented lower weights $(P<0.01)$. The PBS-treated mice had a rapid recurrence of the PMP, with an increase in weight and abdominal volume (Figure 5A). Moreover, all of the PBS-treated mice reached the cutoff weight of $40 \mathrm{~g}$ within the first 4 weeks after resection. The mean $\mathrm{BFV}$ recorded in the SMA increased to $190 \% 4$ weeks after surgery $(P<0.01)$, without any decrease immediately after surgery (Figure $5 \mathrm{~B}$ ). The mean $\mathrm{BFV}$ recorded in the celiac trunk remained unchanged (Figure 5C). In contrast, the bevacizumab-treated mice did not present any increase in their weight or in their abdominal volume until the fourth week (ie, at the end of the follow-up). Moreover, they had lower weights at 4 weeks compared with the untreated mice $(P<0.05)$. At the fourth week, the mean BFVs in the SMA decreased to $75 \%$ of the value recorded before surgery $(P<0.05)$. Moreover, they were lower compared with those recorded in the untreated mice at the fourth week of follow-up $(P<0.001)$ (Figure 5B). The mean BFV remained unchanged in the celiac trunk during the first 3 weeks after surgery and the introduction of anti-angiogenic treatment, but decreased at the fourth week compared with the untreated mice $(P<0.01)$ (Figure 5C). The plasma concentrations of VEGF, PlGF, and TGF- $\beta$ in the mice treated with surgery alone were $178.6 \pm 42.4,12.82 \pm 3.79$, and $65.6 \pm 7.3$ $\mathrm{ng} / \mathrm{L}$, respectively, whereas the levels of these markers decreased in the mice treated with surgery in combination 


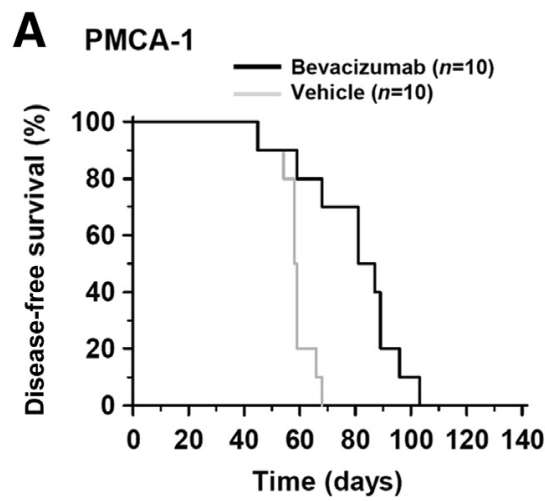

B PMCA-2

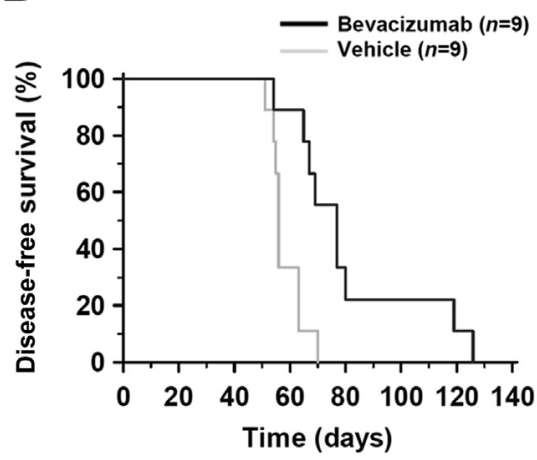

Figure 3 Kaplan-Meier analysis of survival in i.p. tumor-grafted mice injected twice a week with $5 \mathrm{mg} / \mathrm{kg}$ bevacizumab compared with mice injected with vehicle (control mice). A: First model of PMCA (PMCA-1). B: Second model of PMCA (PMCA-2). Mice treated with bevacizumab had a significantly longer survival time than the mice in the control groups in both models. $P<0.001$ (HR $=8.7318$ in $\mathbf{A}$ and $\mathrm{HR}=7.0511$ in $\mathbf{B}$ ).

with bevacizumab to $41.5 \pm 17.4 \mathrm{ng} / \mathrm{L}(P<0.001)$, $4.6 \pm 1.6 \mathrm{ng} / \mathrm{L}(P<0.001)$, and $23.4 \pm 7.0 \mathrm{ng} / \mathrm{L}$ $(P<0.001)$ for VEGF, PlGF, and TGF- $\beta$, respectively. However, the serum levels remained elevated compared with the healthy mice $(P<0.01)$ (Figure 4B).

\section{Discussion}

We established eight reliable and reproducible in vivo models of PMP in immune-compromised nude mice, including one DPAM model and seven PMCA models. The tumors resulting from the i.p. implantation of PMP retained histological, immunohistological, molecular, and topographical characteristics similar to those in humans. The immunostaining analysis highlighted the presence of vessels within the tumor associated with a few isolated $\mathrm{Ki}-67-$ positive tumor cells, thus confirming the low proliferation characteristic of the tumor and this tumor's low sensitivity to standard chemotherapy. ${ }^{26}$

By using our models, we were also able to demonstrate with microangiography and DUS the actual in vivo development of tumor angiogenesis. Moreover, we also demonstrated a correlation between the mean BFV, as measured in the SMA with DUS and the body weight. Finally, we illustrated the antiangiogenic effect of bevacizumab, which manifested as a slower tumor progression.
To our knowledge, our models are among the first mouse models of PMP. We found in the literature only three publications that reported animal models of PMP. ${ }^{15,27,28}$ Flatmark et $\mathrm{al}^{16,27}$ published five mouse models of human PMCA. Indeed, three of the five models consisted of mucinous peritoneal carcinomatosis from various origins. In addition, two of the five models were classified as PMCA-I, according to Ronnett's classification, which corresponded to tumors with a moderate degree of aggressiveness. ${ }^{3}$ In their last study, Flatmark et $\mathrm{al}^{28}$ successfully tested several i.p. chemotherapies and a new immunotoxin immediately after the graft. Although their model is interesting, they i.p. inject mucinous ascites and not solid tumor tissue; therefore, their model is more liquid. Moreover, no study has tested any therapy in an adjuvant setting or after cytoreductive surgery.

Chua et $\mathrm{al}^{15}$ have generated three models of histologically proven DPAM in rats. Although they reported a take rate of $100 \%$ within 3 months after the grafts, they did not provide any information regarding the conservation of their model, which makes their model difficult to reproduce. ${ }^{15}$ In addition, similar

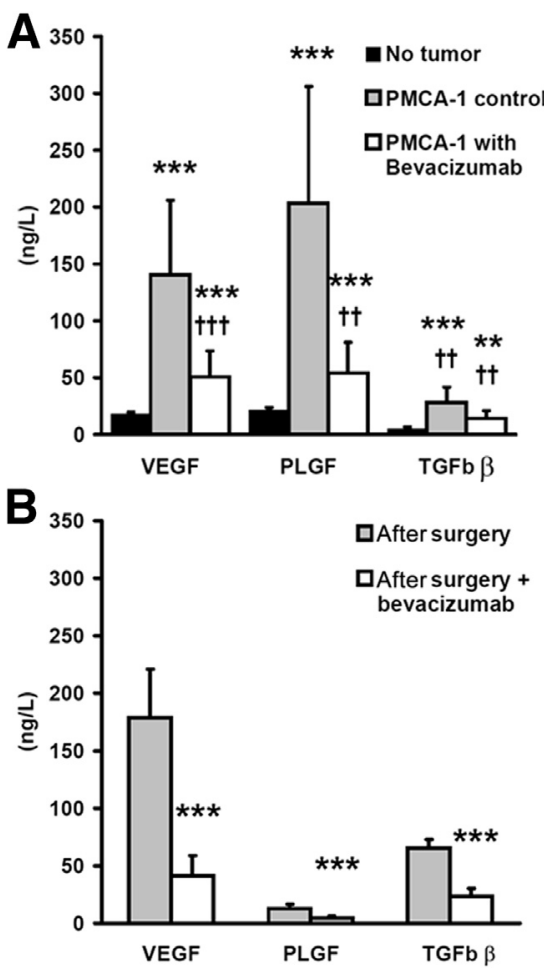

Figure 4 Analysis of serum angiogenesis markers (VEGF, PLGF, and TGF$\beta)$. The expression levels of VEGF, PIGF, and TGF- $\beta$ were significantly increased in PMP-grafted mice $(n=5)$ compared with healthy mice $(n=$ 5). A: The expression levels were decreased in the PMP-grafted mice that were injected twice a week with $5 \mathrm{mg} / \mathrm{kg}$ bevacizumab $(n=5)$, but remained elevated compared with the control group. Analysis of serum angiogenesis markers (VEGF, PLGF, and TGF- $\beta$ ) in healthy mice $(n=5)$, in PMP-grafted mice treated with cytoreductive surgery and PBS $(n=9)$, and in grafted mice treated with cytoreductive surgery and $5 \mathrm{mg} / \mathrm{kg}$ bevacizumab injected twice a week $(n=11)$. B: The expression levels were decreased in the bevacizumab-treated group, but remained elevated compared with the control group. ${ }^{* *} P<0.01,{ }^{* * *} P<0.001$ versus healthy mice; ${ }^{\dagger \dagger} P<0.01,{ }^{\dagger \dagger} P<0.001$ versus the vehicle group. 
to Flatmark et al, ${ }^{27}$ the models of Chua et $\mathrm{al}^{5}$ corresponded to a benign subtype of PMP, precluding the meaningful assessment of anti-angiogenic treatments. The histopathological subtype of a tumor is an independent predictor of poor progression-free survival and poor overall survival. Consequently, the assessment of anti-angiogenic drugs should be performed on the most
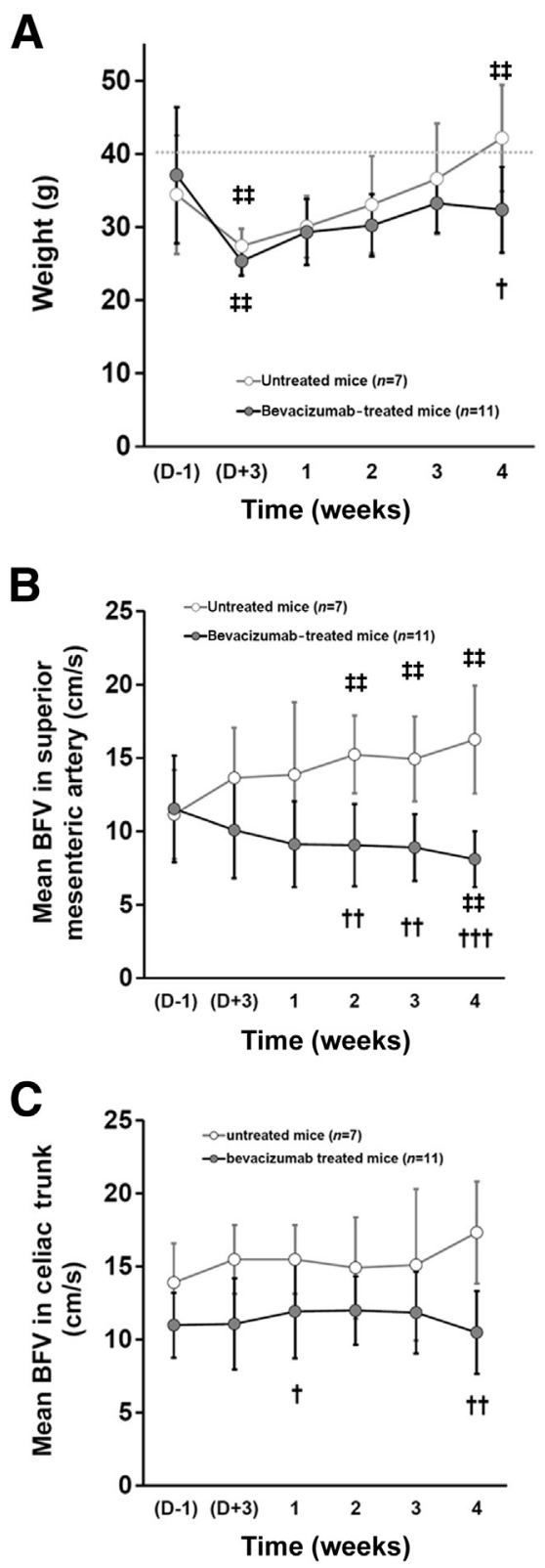

Figure 5 Treatment with an anti-angiogenic agent (bevacizumab) after cytoreductive surgery. All of the mice had reached 35 to $40 \mathrm{~g}$ before surgery. After resection, the weight decreased significantly in both groups. A: In the PBS-treated group, the weight was significantly higher at 4 weeks compared with the preresection weight, whereas no significant difference was found in the bevacizumab-treated group. B: In the PBS-treated group, the mean BFV in the SMA increased progressively after surgery, whereas the BFV decreased in the bevacizumab-treated group. C: In the PBS-treated group, the mean BFV in the celiac trunk remained stable after surgery, whereas the BFV decreased at the fourth week in the bevacizumab-treated group. ${ }^{\ddagger \ddagger} P<0.01$ versus the preresection weight. ${ }^{\dagger} P<0.05,{ }^{\dagger \dagger} P<0.01$, and ${ }^{\dagger \dagger} P<0.001$ versus PBS-treated mice. aggressive models of PMP. In our study, we developed PMCA animal models of the most aggressive subtypes, which are the most useful for evaluating anti-angiogenic treatments.

Angiogenesis has become a new area for intensive research in imaging. The quantification of angiogenesis requires new models and more refined tools or the implementation of functional applications of well-established techniques for monitoring the response to newly developed anti-angiogenic drugs. The development of techniques to quantify tissue activity may help predict tumor sensitivity to a specific treatment and allow for the early detection of the absence of a response to new and expensive therapies that are not free from adverse effects.

Noninvasive functional imaging modalities, such as magnetic resonance imaging, dynamic contrast-enhanced computed tomography, and positron emission tomography, have been used for the assessment of the vessels and microcirculation in animals. However, the limitations of these techniques include a high cost and limited availability. For preclinical studies in small animals, we require noninvasive methods that ideally would be reliable, rapid, reproducible, easy to repeat during a prolonged follow-up, and affordable. Two-dimensional, color-coded, pulsed DUS for the measurement of BFV in tumor-feeding vessels provides a powerful tool for the in vivo evaluation of tumor growth and the assessment of neovascularization in preclinical studies and can be a useful technique to assess treatment efficacy. ${ }^{19-21}$

We used the SMA mean BFV recordings to assess the functionality and density of the downstream vessels. In our study, we found greater mean BFVs in the SMAs of mice with peritoneal tumors containing abnormal vessels. The peritoneal vasculature is mainly supplied by the SMA. According to our results, mean BFV measurements in the SMA could be an indirect indication of the development of vessels in the peritoneal tumor and constitute a useful tool for the assessment of neovascularization in PMP in mice. The results of the DUS, along with an increased abnormal vessel density observed with microangiography and an increase in the blood markers of angiogenesis, reinforce our hypothesis that our orthotopic murine models of PMP are relevant to test angiogenesis and the putative beneficial effects of anti-angiogenic therapies.

The potentially beneficial effects of anti-angiogenic therapies have led to the development of various drugs that require preclinical studies in animals to evaluate their efficacy and toxicity before clinical trials can be initiated. The role of angiogenesis and vascular remodeling in the development of cancer has been largely demonstrated. ${ }^{29,30}$ Consequently, tumor vessels have become an important target in cancer therapy with the development of novel targeted therapies. ${ }^{31-33}$ However, these clinical developments appear to have more challenges to controlling tumor growth, such that preclinical studies in small animals with the evaluation of tumor angiogenesis are required. We postulated that PMP models could be used for testing the efficacy of new anti-angiogenic drugs because PMP tumors grow slowly. Because of the associated antitumor effect of most anti-angiogenic agents, testing only the anti-angiogenic function, apart from the antitumor 
function, is difficult with common cancers. Indeed, the low proliferative profile of PMP associated with neo-angiogenesis allows for the evaluation of the efficacy of anti-angiogenic drugs without any bias induced by an anti-proliferative effect. PMP is a rare disease, but this animal model could offer a new possibility to replace the Matrigel test that is usually proposed. Indeed, bevacizumab induced a significant delay in tumor growth, according to our experiments.

Recent studies have revealed that anti-VEGF therapy may have a limited effect on tumor vessels,${ }^{34-36}$ highlighting the importance of developing other anti-angiogenic strategies for cancer treatment. Other therapeutic targets may be explored through identifying cytotoxic drugs that target the atypical cells or disturb the mucinous excretion component of the tumor cells. More important, there is a need to recognize why conventional DNA-damaging cytotoxic drugs have a limited efficacy in PMP. Indeed, most PMPs contain large amounts of mucin but are paucicellular. Antiinflammatory drugs could inhibit extracellular mucin production in PMP by targeting inflammatory cascades and may increase the disease-free interval or reduce the extent or frequency of morbid cytoreductive surgical procedures. ${ }^{37}$

Our study has several limitations. First, in xenograft models, tumor vessel formation originates from the host mouse. The loss of human stromal cells was reported in a large colon cancer xenograft panel that was recently published.$^{38}$ For this reason, it could be postulated that some drugs may be ineffective, or less effective, because the drugs are targeted to human vessels and not to mouse vessels. Although theoretically possible, we did not observe such an occurrence in our models. Second, DUS is an operator-dependent technique. Therefore, sampling of the mean BFV was repeated five times and averaged to limit intraobserver variability. Moreover, the investigator performing the DUS (P.B.) was blinded to the treatment for each mouse. Third, although we demonstrated angiogenesis in PMP, because of the poor cellularity inside the tumor and the amount of nonvascularized mucinous component, this angiogenesis does not account for the global increase in blood flow in the SMA. However, isolectin microangiography indicated irregular and tortuous neovessels in the tumor. It is likely that further experimentation with in vivo functional imaging of the tumor will help to understand the changes in tumor vascularity in terms of permeability and changes in the size of the vessels.

In conclusion, we established eight reproducible murine models of PMP, which are similar to human PMP, particularly regarding the local peritoneal development and the absence of metastasis. Moreover, the human histological features of the tumor are retained in the mouse. In this model, we highlighted the role of tumor angiogenesis, even for this paucicellular tumor. The anti-angiogenic drug (bevacizumab) has proven benefit with respect to the reduction of tumor microvessel density, as revealed with DUS and the normalization of the microvessels and when this drug is introduced as an adjuvant therapy after cytoreductive surgery. This promising adjuvant therapy will help to avoid a recurrence of the disease and the re-accumulation of mucin, which characterizes the failure of surgical treatments and is responsible for repeated treatment and increased morbidity.

\section{Acknowledgments}

We thank Dr. Raphaëlle Audollent and Aurore Rampanou for help with experiments, Drs. Isabelle Madelaine and Nathalie Jourdan for technical support, and Dr. Judith Nemeth for histopathological analysis.

\section{Supplemental Data}

Supplemental material for this article can be found at http://dx.doi.org/10.1016/j.ajpath.2014.03.004.

\section{References}

1. Cuatrecasas M, Matias-Guiu X, Prat J: Synchronous mucinous tumors of the appendix and the ovary associated with pseudomyxoma peritonei: a clinicopathologic study of six cases with comparative analysis of c-Ki-ras mutations. Am J Surg Pathol 1996, 20:739-746

2. Guerrieri C, Franlund B, Fristedt S, Gillooley JF, Boeryd B: Mucinous tumors of the vermiform appendix and ovary, and pseudomyxoma peritonei: histogenetic implications of cytokeratin 7 expression. Hum Pathol 1997, 28:1039-1045

3. Ronnett BM, Shmookler BM, Diener-West M, Sugarbaker PH, Kurman RJ: Immunohistochemical evidence supporting the appendiceal origin of pseudomyxoma peritonei in women. Int J Gynecol Pathol 1997, 16:1-9

4. Szych C, Staebler A, Connolly DC, Wu R, Cho KR, Ronnett BM: Molecular genetic evidence supporting the clonality and appendiceal origin of Pseudomyxoma peritonei in women. Am J Pathol 1999, 154:1849-1855

5. Chua TC, Moran BJ, Sugarbaker PH, Levine EA, Glehen O, Gilly FN, Baratti D, Deraco M, Elias D, Sardi A, Liauw W, Yan TD, Barrios P, Gomez Portilla A, de Hingh IH, Ceelen WP, Pelz JO, Piso P, GonzalezMoreno S, Van Der Speeten K, Morris DL: Early- and long-term outcome data of patients with pseudomyxoma peritonei from appendiceal origin treated by a strategy of cytoreductive surgery and hyperthermic intraperitoneal chemotherapy. J Clin Oncol 2012, 30:2449-2456

6. Elias D, Gilly F, Quenet F, Bereder JM, Sideris L, Mansvelt B, Lorimier G, Glehen O: Pseudomyxoma peritonei: a French multicentric study of 301 patients treated with cytoreductive surgery and intraperitoneal chemotherapy. Eur J Surg Oncol 2010, 36:456-462

7. Miner TJ, Shia J, Jaques DP, Klimstra DS, Brennan MF, Coit DG: Long-term survival following treatment of pseudomyxoma peritonei: an analysis of surgical therapy. Ann Surg 2005, 241:300-308

8. Moran B, Baratti D, Yan TD, Kusamura S, Deraco M: Consensus statement on the loco-regional treatment of appendiceal mucinous neoplasms with peritoneal dissemination (pseudomyxoma peritonei). J Surg Oncol 2008, 98:277-282

9. Smeenk RM, Verwaal VJ, Antonini N, Zoetmulder FA: Progression of pseudomyxoma peritonei after combined modality treatment: management and outcome. Ann Surg Oncol 2007, 14:493-499

10. Sugarbaker PH: New standard of care for appendiceal epithelial neoplasms and pseudomyxoma peritonei syndrome? Lancet Oncol 2006, 7:69-76

11. Yan TD, Chu F, Links M, Kam PC, Glenn D, Morris DL: Cytoreductive surgery and perioperative intraperitoneal chemotherapy for peritoneal carcinomatosis from colorectal carcinoma: non-mucinous tumour associated with an improved survival. Eur J Surg Oncol 2006, 32:1119-1124

12. Ronnett BM, Yan H, Kurman RJ, Shmookler BM, Wu L, Sugarbaker PH: Patients with pseudomyxoma peritonei associated with disseminated peritoneal adenomucinosis have a significantly more favorable prognosis than patients with peritoneal mucinous carcinomatosis. Cancer 2001, 92:85-91 
13. Sun WL, Hutarew G, Gradl J, Gratzl M, Denz H, Fiegl M: Successful antiangiogenic combination therapy for pseudomyxoma peritonei with bevacizumab and capecitabine. Cancer Biol Ther 2009, 8:1459-1462

14. Winer I, Buckanovich RJ: Bevacizumab for the treatment of nonresectable Pseudomyxoma peritonei associated with mucinous ovarian tumor of low malignant potential: a comparison of two cases. Case Rep Oncol 2009, 3:1-8

15. Chua TC, Akther J, Yao P, Morris DL: In vivo model of pseudomyxoma peritonei for novel candidate drug discovery. Anticancer Res 2009, 29:4051-4055

16. Flatmark K, Davidson B, Kristian A, Stavnes HT, Forsund M, Reed W: Exploring the peritoneal surface malignancy phenotype: a pilot immunohistochemical study of human pseudomyxoma peritonei and derived animal models. Hum Pathol 2010, 41:1109-1119

17. Berge M, Bonnin P, Sulpice E, Vilar J, Allanic D, Silvestre JS, Levy BI, Tucker GC, Tobelem G, Merkulova-Rainon T: Small interfering RNAs induce target-independent inhibition of tumor growth and vasculature remodeling in a mouse model of hepatocellular carcinoma. Am J Pathol 2010, 177:3192-3201

18. Delorme S, Haberkorn U, Kinscherf R, Zuna I, Bahner ML, van Kaick G: Changes of tumor vascularity during gene therapy monitored with color Doppler US. Ultrasound Med Biol 2001, 27:1595-1603

19. Eveno C, Le Henaff C, Audollent R, Soyer P, Rampanou A, Nemeth J, Brouland JP, Dupuy E, Pocard M, Bonnin P: Tumor and non-tumor liver angiogenesis is traced and evaluated by hepatic arterial ultrasound in murine models. Ultrasound Med Biol 2012, 38:1195-1204

20. Van de Veire S, Stalmans I, Heindryckx F, Oura H, TijerasRaballand A, Schmidt T, et al: Further pharmacological and genetic evidence for the efficacy of PlGF inhibition in cancer and eye disease. Cell 2010, 141:178-190

21. Vincent F, Bonnin P, Clemessy M, Contreres JO, Lamande N, Gasc JM, Vilar J, Hainaud P, Tobelem G, Corvol P, Dupuy E: Angiotensinogen delays angiogenesis and tumor growth of hepatocarcinoma in transgenic mice. Cancer Res 2009, 69:2853-2860

22. Bonnin P, Villemain A, Vincent F, Debbabi H, Silvestre JS, Contreres JO, Levy BI, Tobelem G, Dupuy E: Ultrasonic assessment of hepatic blood flow as a marker of mouse hepatocarcinoma. Ultrasound Med Biol 2007, 33:561-570

23. Flaman J-M, Frebourg T, Moreau V, Charbonnier F, Martin C, Chappuis P, Sappino A-P, Limacher I-M, Bron L, Benhattar J, Tada M, Van Meir EG, Estreicher A, Iggo RD: A simple p53 functional assay for screening cell lines, blood, and tumors. Proc Natl Acad Sci U S A 1995, 92:3963-3967

24. Lievre A, Bachet JB, Boige V, Cayre A, Le Corre D, Buc E, Ychou M, Bouche O, Landi B, Louvet C, Andre T, Bibeau F, Diebold MD, Rougier P, Ducreux M, Tomasic G, Emile JF, Penault-Llorca F, Laurent-Puig P: KRAS mutations as an independent prognostic factor in patients with advanced colorectal cancer treated with cetuximab. J Clin Oncol 2008, 26:374-379
25. Goldstein IJ, Winter HG: The Griffonia simplicifolia I-B4 isolectin: a probe for alpha-D-galactosyl end groups. Subcell Biochem 1999, 32:127-141

26. Nakakura EK: Pseudomyxoma peritonei: more questions than answers. J Clin Oncol 2012, 30:2429-2430

27. Flatmark K, Reed W, Halvorsen T, Sorensen O, Wiig JN, Larsen SG, Fodstad O, Giercksky KE: Pseudomyxoma peritonei: two novel orthotopic mouse models portray the PMCA-I histopathologic subtype. BMC Cancer 2007, 7:116

28. Flatmark K, Guldvik IJ, Svensson H, Fleten KG, Ann Florenes V, Reed W, Giercksky KE, Fodstad O, Andersson Y: Immunotoxin targeting EpCAM effectively inhibits peritoneal tumor growth in experimental models of mucinous peritoneal surface malignancies. Int J Cancer 2013, 133:1497-1506

29. Carmeliet P, Jain RK: Angiogenesis in cancer and other diseases. Nature 2000, 407:249-257

30. Folkman J: Angiogenesis-dependent diseases. Semin Oncol 2001, 28: $536-542$

31. Davis DW, McConkey DJ, Abbruzzese JL, Herbst RS: Surrogate markers in antiangiogenesis clinical trials. Br J Cancer 2003, 89:8-14

32. Fox SB, Gasparini G, Harris AL: Angiogenesis: pathological, prognostic, and growth-factor pathways and their link to trial design and anticancer drugs. Lancet Oncol 2001, 2:278-289

33. Scappaticci FA: Mechanisms and future directions for angiogenesisbased cancer therapies. J Clin Oncol 2002, 20:3906-3927

34. Helfrich I, Scheffrahn I, Bartling S, Weis J, von Felbert V, Middleton M, Kato M, Ergun S, Schadendorf D: Resistance to antiangiogenic therapy is directed by vascular phenotype, vessel stabilization, and maturation in malignant melanoma. J Exp Med 2010, 207:491-503

35. Huang D, Ding Y, Zhou M, Rini BI, Petillo D, Qian CN, Kahnoski R, Futreal PA, Furge KA, Teh BT: Interleukin-8 mediates resistance to antiangiogenic agent sunitinib in renal cell carcinoma. Cancer Res 2010, 70:1063-1071

36. Priceman SJ, Sung JL, Shaposhnik Z, Burton JB, Torres-Collado AX, Moughon DL, Johnson M, Lusis AJ, Cohen DA, Iruela-Arispe ML, Wu L: Targeting distinct tumor-infiltrating myeloid cells by inhibiting CSF-1 receptor: combating tumor evasion of antiangiogenic therapy. Blood 2010, 115:1461-1471

37. Choudry HA, Mavanur A, O'Malley ME, Zeh HJ, Guo Z, Bartlett DL: Chronic anti-inflammatory drug therapy inhibits gel-forming mucin production in a murine xenograft model of human pseudomyxoma peritonei. Ann Surg Oncol 2012, 19:1402-1409

38. Julien S, Merino-Trigo A, Lacroix L, Pocard M, Goere D, Mariani P, Landron S, Bigot L, Nemati F, Dartigues P, Weiswald LB, Lantuas D, Morgand L, Pham E, Gonin P, Dangles-Marie V, Job B, Dessen P, Bruno A, Pierre A, De The H, Soliman H, Nunes M, Lardier G, Calvet L, Demers B, Prevost G, Vrignaud P, Roman-Roman S, Duchamp O, Berthet C: Characterization of a large panel of patientderived tumor xenografts representing the clinical heterogeneity of human colorectal cancer. Clin Cancer Res 2012, 18:5314-5328 